\title{
ON THE NUMERATORS OF THE CONVERGENTS OF THE STIELTJES CONTINUED FRACTIONS*
}

\author{
BY \\ JACOB SHERMAN
}

Introduction. The object of this paper is the study of the numerators of the infinite continued fractions introduced by Stieltjes $[1,2] . \dagger$ They are of two types:

(a) the "associated" continued fraction:

$$
K(z) \equiv \frac{\lambda_{1} \mid}{\mid z-c_{1}}-\frac{\lambda_{2} \mid}{\mid z-c_{2}}-\frac{\lambda_{3} \mid}{\mid z-c_{3}}-\cdots,
$$

the $n$th convergent of which will be denoted by $\Omega_{n}(z) / \phi_{n}(z) \equiv K_{n}(z)(n=0$, $1,2,3, \cdots)$;

(b) the "corresponding" continued fraction:

$$
W(z) \equiv \frac{b_{1} \mid}{\mid z}-\frac{b_{2} \mid}{\mid 1}-\frac{b_{3} \mid}{\mid z}-\frac{b_{4} \mid}{\mid 1}-\cdots,
$$

the $n$th convergent of which will be denoted by $U_{n}(z) / V_{n}(z) \equiv W_{n}(z)(n=0$, $1,2,3, \cdots)$. In (a) $\lambda_{i}, c_{i}$ are real constants with $\lambda_{i}>0$ for $i=1,2,3, \ldots$; $\Omega_{n}(z), \phi_{n}(z)$ are polynomials of degree $n-1$ and $n$ respectively. $\neq$ In (b) $b_{i}$ are real constants, $b_{1}>0, b_{2 i+1} b_{2 i}>0$ for $i=1,2, \cdots U_{2 n+\epsilon}(z), V_{2 n+\epsilon}(z)$ are polynomials of degree $(n+\epsilon-1)$ and $(n+\epsilon)$ respectively, where $\epsilon=0,1$. For our study we shall need certain results from the theory of continued fractions [2] and of the so-called moments problem [3].

(i) The convergence of the associated and corresponding continued fractions. Here of fundamental importance is Grommer's Selection Theorem [2]:

From every sequence of convergents of a continued fraction of type (1) or (2) there may be selected a sub-sequence, which for all non-real $z$ converges to $a$ Stieltjes integral of the form $\int_{-\infty}^{\infty}(1 /(z-u)) d \psi(u)$.

Here $\psi(z)$ (and hereafter $\psi_{1}(z), \psi_{2}(z), \cdots$ ) denotes generally a bounded monotonic non-decreasing function with infinitely many points of increase in $(-\infty, \infty)$, such that all integrals $\int_{-\infty}^{\infty} z^{n} d \psi(z) \equiv \alpha_{n}$ ("moments") exist (for

* Presented to the Society, March 26, 1932; received by the editors April 28, 1932.

The author wishes to express his gratitude to Professor J. Shohat for many valuable suggestions concerning the subject of this paper.

$\dagger$ The numbers in brackets refer to the list of literature at the end of the paper.

$\ddagger$ We note that $\Omega_{n}(z) \equiv \lambda_{1} z^{n-1}+\cdots$ does not depend on $\lambda_{2}$ and $\phi_{n}(z) \equiv z^{n}+\cdots$ does not depend on $\lambda_{1}$. This will be useful in our later discussion. 
$n=0,1,2,3, \cdots)$, with $\alpha_{0}>0$. We may without loss of generality assume $\psi(-\infty)=0$. The continued fraction (1) or (2) is said to be "associated" with or "corresponding" to, respectively, the integral $\int_{-\infty}^{\infty}(1 /(z-u)) d \psi(u)$ or its formal development

$$
P\left(\frac{1}{z}\right)=\sum_{n=0}^{\infty} \frac{\alpha_{n}}{z^{n+1}}
$$

In symbols:

$$
\begin{array}{r}
F(z) \equiv \int_{-\infty}^{\infty} \frac{d \psi(u)}{z-u}=P\left(\frac{1}{z}\right) \equiv \sum_{n=0}^{\infty} \frac{\alpha_{n}}{z^{n+1}} \sim \frac{\lambda_{1} \mid}{\mid z-c_{1}}-\frac{\lambda_{2} \mid}{\mid z-c_{2}}-\cdots \\
\quad\left(\int_{-\infty}^{\infty} z^{n} d \psi(z)=\alpha_{n}\right) .
\end{array}
$$

The association (3) means formally [3]

$$
\int_{-\infty}^{\infty} \frac{d \psi(u)}{z-u}-K_{n}(z)=\frac{\alpha^{\prime}}{z^{2 n+1}}+\frac{\alpha^{\prime \prime}}{z^{2 n+2}}+\cdots ;
$$

similarly the correspondence means formally

$$
\int_{-\infty}^{\infty} \frac{d \psi(u)}{z-u}-W_{n}(z)=\frac{\beta^{\prime}}{z^{n+1}}+\frac{\beta^{\prime \prime}}{z^{n+2}}+\cdots .
$$

It is known that a continued fraction of form (1) may be obtained from one of form (2) by "contraction," and then

$$
A_{2 n}(z) \equiv \Omega_{n}(z), \quad B_{2 n}(z) \equiv \phi_{n}(z) \quad(n=0,1,2,3, \cdots) .
$$

The "association" (4) shows [2] that the $\left\{\phi_{n}(z)\right\}^{*}$ constitute an orthogonal set of polynomials with regard to the distribution $d \psi(x)$, i.e.

$$
\int_{-\infty}^{\infty} \phi_{m}(x) \phi_{n}(x) d \psi(x)=\left\{\begin{array}{ll}
0, & m \neq n, \\
>0, & m=n
\end{array} \quad(m, n=0,1,2,3, \cdots),\right.
$$

where $(-\infty, \infty)$ may be "reducible" (see page 70) to a sub-interval $(a, b)$. We might have $\psi(x)=\int_{-\infty}^{x} p(x) d x(p(x) \geqq 0$ in $(-\infty, \infty))$; then in all our formulas $d \psi(x)$ is to be replaced by $p(x) d x$. In particular (5) becomes

$$
\int_{-\infty}^{\infty} \phi_{m}(x) \phi_{n}(x) p(x) d x=0, m \neq n \quad(m, n=0,1,2,3, \cdots) .
$$

Here we say that $\left\{\phi_{n}(x)\right\} \equiv\left\{\phi_{n}(x ; a, b ; p)\right\}$ forms an orthogonal set corresponding to the characteristic function $p(x)(p(x) \geqq 0)$ in $(-\infty, \infty))$. In some

* Throughout this paper $z$ represents the complex variable $x+i y$. 
instances we might have $\psi(x)=\psi(a)$ for $x<a$ and $\psi(x)=\psi(b)$ for $x>b$ $(p(x) \equiv 0$ outside $(a, b))$ in which case $\int_{-\infty}^{\infty}(1 /(z-u)) d \psi(u)$ reduces to $\int_{a}^{b}(1 /(z-u)) d \psi(u)$ and (5) becomes

$$
\int_{a}^{b} \phi_{m}(x) \phi_{n}(x) d \psi(x)=0, m \neq n(m, n=0,1,2,3, \cdots) .
$$

The function $F(z) \equiv \int_{-\infty}^{\infty}(1 /(z-u)) d \psi(u)$ in (3) is known [2] to be regular and analytic in any finite closed region of the complex $z$-plane which does not contain any portion of the real axis. Such a region we call, for brevity, an " $\Omega$-region" [3]. In such a region $1 / F(z)$ is also analytic. In fact it is readily proved that $F(z)$ has no zeros in an $\Omega$-region. We now introduce, following Hamburger [3], the continued fraction

$$
K_{n}(z, t) \equiv \frac{\lambda_{1} \mid}{\mid z-c_{1}}-\frac{\lambda_{2} \mid}{\mid z-c_{2}}-\cdots-\frac{\lambda_{n-1} \mid}{\mid z-c_{n-1}}-\frac{\lambda_{n} \mid}{\mid z-c_{n}+t},
$$

$\lambda_{i}, c_{i}$ as in (1), $t$ real, arbitrary; $K_{n}(z, \infty) \equiv K_{n-1}(z)$. Evidently $K_{n}(z, 0)$ $\equiv K_{n}(z)$.

We take, from Hamburger [3], the following

Definition. The continued fraction $K(z)$ converges "completely" to the function $F(z)$ of the type (3) if, for arbitrarily small $\epsilon>0$ and for every $\Omega$-region, $\left|K_{n}(z, t)-F(z)\right|<\epsilon(z$ in $\Omega, n \geqq N)$ for every real arbitrary $t$, including $t=\infty$, where $N$ depends on $\epsilon$ and $\Omega$ only.

(ii) The $\left[\alpha_{n}\right]_{0}^{\infty}$-moments problem. By this is meant the determination of $\psi(z)$ of the above nature, given the real set $\left[\alpha_{n}\right](n=0,1,2, \cdots)$ of its moments. If the set determines $\psi(z)$ uniquely (disregarding additive constants) the moments problem is said to be "determined"; otherwise it is said to be "indetermined." In case of an "association," as given in (4), we say that $\psi(z)$ is the solution of the moments problem related to $P(1 / z)$ or $K(z)$, or, see (5), to the orthogonal set $\left\{\phi_{n}(x)\right\}$. Hamburger [3] has shown that the complete convergence of the associated continued fraction $K(z)$ to the integral $\int_{-\infty}^{\infty}(1 /(z-u)) d \psi(u)$ is a necessary and sufficient condition for the moments problem related to $K(z)$ to be determined over the interval $(-\infty$, $\infty)$.

Our purpose is the study of the polynomials $\left\{U_{2 n}(x)\right\},\left\{U_{2 n+1}(x)\right\}$, $\left\{V_{2 n+1}(x)\right\}$ and their relations to the orthogonal polynomials $\left\{\phi_{n}(x)\right\}$. Some of the results concerning $\left\{U_{2 n}(x)\right\}$ have been presented by J. Shohat and J. Sherman in [4]. 
1. The orthogonality properties of the numerators $\Omega_{n}(x)$. The study of the $\Omega_{n}(x)$ is based upon the continued fraction

$$
K^{\prime}(z) \equiv \frac{\lambda_{2} \mid}{\mid z-c_{2}}-\frac{\lambda_{3} \mid}{\mid z-c_{3}}-\cdots
$$

$\left(\lambda_{i}, c_{i}\right.$ the same as in (1)), whose successive convergents will be denoted by $P_{n}(z) / Q_{n}(z) \equiv K_{n}^{\prime}(z)(n=0,1,2,3, \cdots)$.

LEMMA 1. $\Omega_{n+1}(z)=\lambda_{1} Q_{n}(z) \cdot(n \geqq 0)$.

We have for $K(z), K^{\prime}(z)$ respectively

$$
\begin{array}{r}
\Omega_{n+1}(z)=\left(z-c_{n+1}\right) \Omega_{n}(z)-\lambda_{n+1} \Omega_{n-1}(z) \quad(n=1,2,3, \cdots) \\
\left(\Omega_{0}(z) \equiv 0, \Omega_{1}(z)=\lambda_{1}, \Omega_{2}(z)=\lambda_{1}\left(z-c_{2}\right)\right) ; \\
Q_{n}(z)=\left(z-c_{n+1}\right) Q_{n-1}(z)-\lambda_{n+1} Q_{n-2}(z) \quad(n=2,3,4, \cdots) \\
\left(Q_{0}(z) \equiv 1, Q_{1}(z)=z-c_{2}\right) .
\end{array}
$$

The truth of the lemma is seen by comparing (8) and (9). In fact, they represent the same difference equation with the initial conditions $Q_{0}(z), Q_{1}(z)$ and $\Omega_{1}(z), \Omega_{2}(z)$, respectively, differing only by a constant factor $\left(\lambda_{1}\right)$. Our lemma leads to the following important conclusion:

$K^{\prime}(z)$ is an infinite continued fraction, the denominators of whose convergents are, to within a constant factor independent of $n$, identical with the numerators of the convergents of $K(z)$.

Lемма 2. $K^{\prime}(z)$ is associated with one and only one "positive definite" power series.*

In symbols

$$
K^{\prime}(z) \sim P_{1}(1 / z) \equiv \sum_{n=0}^{\infty} \frac{\beta_{n}}{z^{n+1}}
$$

This follows directly from the results due to Hamburger [3], since, in $K^{\prime}(z)$, all $\lambda_{i}>0(i=1,2,3, \cdots)$.

LEMмA 3. If $K(z)$ converges in a certain $\Omega$-region, then $K^{\prime}(z)$ converges in that same region to a function which is regular and analytic in that region.

* The series

$$
P(1 / z) \equiv \sum_{n=0}^{\infty} \frac{\alpha_{n}}{z^{n+1}}
$$

is said to be "positive definite" if all the determinants

$$
\Delta_{m} \equiv\left[\alpha_{i, j}\right]_{i, j=0}^{m-1}>0 \text { for } m=1,2, \cdots,
$$

where $\alpha_{i, j}=\alpha_{i+j}$. We set also $\Delta_{0} \equiv 1$. 
It is known [2] that, if $K(z)$ is convergent, $K^{\prime}(z)$ must either converge or diverge to infinity. The latter is impossible, for, by Grommer's Theorem, we may select a sub-sequence $\left\{K_{n_{i}^{\prime}}(z)\right\}(i=1,2,3, \cdots)$ of the convergents of $K^{\prime}(z)$ which in the $\Omega$-region converges to a Stieltjes integral of the form (3):

$$
F_{1}(z) \equiv \int_{-\infty}^{\infty} \frac{d \psi_{1}(u)}{z-u}
$$

and $F_{1}(z)$ is regular and analytic in $\Omega$. Hence $K^{\prime}(z)$ itself converges in that $\Omega$-region to $F_{1}(z)$.

CoROLLARY. If $F(z)$ and $F_{1}(z)$, both analytic and non-vanishing in $\Omega$, are the limit functions of $K(z)$ and $K^{\prime}(z)$ respectively, then

$$
F(z)=\frac{\lambda_{1}}{z-c_{1}-F_{1}(z)}
$$

$(z$ in $\Omega)$.

LEMma 4. If $K(z)$ converges completely to

$$
F(z) \equiv \int_{-\infty}^{\infty} \frac{d \psi(u)}{z-u}
$$

then $K^{\prime}(z)$ converges completely to a certain

$$
F_{1}(z) \equiv \int_{-\infty}^{\infty} \frac{d \psi_{1}(u)}{z-u}
$$

By the definition of $K_{n}(z, t)$ (see (6)) we write

$$
\begin{aligned}
& K_{n}(z, t)=\frac{\lambda_{1}}{z-c_{1}-K_{n-1}^{\prime}(z, t)} \\
& \left(K_{n-1}^{\prime}(z, t) \equiv \frac{\lambda_{2} \mid}{\mid z-c_{2}}-\frac{\lambda_{3} \mid}{\mid z-c_{3}}-\cdots-\frac{\lambda_{n-1} \mid}{\mid z-c_{n-1}}-\frac{\lambda_{n} \mid}{\mid z-c_{n}+t}\right) .
\end{aligned}
$$

Hence $K_{n-1}^{\prime}(z, t)$ plays the same rôle for $K^{\prime}(z)$ as $K_{n-1}(z, t)$ does for $K(z)$. Using the definition of complete convergence,

$$
\left|K_{n}(z, t)-F(z)\right|<\epsilon \quad(n \geqq N(\epsilon, \Omega), z \text { in } \Omega),
$$

we are lead through (10) and (11), and with the same $z, n, \epsilon, t, \Omega$ as in (12), to

$$
\left|K_{n-1}^{\prime}(z, t)-F_{1}(z)\right| \leqq \frac{\epsilon}{\lambda_{1}} \cdot\left|\frac{\lambda_{1}}{K_{n}(z, t)}\right| \cdot\left|\frac{\lambda_{1}}{F(z)}\right| .
$$

Furthermore, we know that 


$$
\begin{aligned}
\lambda_{1}>0 ;|F(z)| \geqq h>0 & (z \text { in } \Omega), \\
\left|K_{n}(z, t)\right| \geqq \frac{h}{2}>0 & (z \text { in } \Omega, n \geqq N)
\end{aligned}
$$

Hence

$$
\frac{1}{\lambda_{1}}\left|\frac{\lambda_{1}}{K_{n}(z, t)}\right| \cdot\left|\frac{\lambda_{1}}{F(z)}\right|
$$

is bounded above for all real $t$ (including $t=\infty$ ) and for all $z$ in $\Omega$, and thus our lemma is proved.

Combining our lemmas, we are in a position to prove

ThEOREM I. The numerators $\left\{\Omega_{n}(x)\right\}$ of the continued fraction $K(x)$ or, which is the same, the numerators of the even convergents of the continued fraction $W(x)$, constitute a set of polynomials orthogonal with regard to a function $\psi_{1}(x)$ of the same type as $\psi(x)$, i.e.

$$
\int_{-\infty}^{\infty} \Omega_{m}(x) \Omega_{n}(x) d \psi_{1}(x)=0, m \neq n \quad(m, n=1,2,3, \cdots) .
$$

TheOREM II. $\psi_{1}(z)$, as a solution of the moments problem associated with $K^{\prime}(z)$, is uniquely determined if $\psi(z)$ is uniquely determined as a solution of the moments problem associated with $K(z)$.

The proof of Theorem I follows from the fact that $K^{\prime}(z)$, being of the same type as $K(z)$, is, therefore, associated, in the sense of (4), with at least one Stieltjes integral of type $(3): \int_{-\infty}^{\infty}(1 /(z-u)) d \psi_{1}(u)$, which leads to orthogonality relations (13) similar to $(5)$, and here also $(-\infty, \infty)$ may reduce to a certain sub-interval.

Theorem II follows from Lemma 4 combined with Hamburger's necessary and sufficient condition for the determined character of the moments problem as given above (see page 66).

We can also state

TheOREM III. Let $(\lambda, L)$ and $\left(\lambda^{\prime}, L^{\prime}\right)$ denote the "true" intervals of orthogonality for $\left\{\phi_{n}(x)\right\}$ and $\left\{\Omega_{n}(x)\right\}$ respectively.

(i) $\left(\lambda^{\prime}, L^{\prime}\right) \subset(\lambda, L)$.

(ii) If $(\lambda, L)$ is finite, then, in general, $\lambda^{\prime}=\lambda$ and $L^{\prime}=L$. Any sub-interval of $(\lambda, L)$ which does not contain an interval of constancy of $\psi(x)$ possesses the same property with regard to $\psi_{1}(x)$.

(iii) If $(\lambda, L)$ is infinite, so is $\left(\lambda^{\prime}, L^{\prime}\right)$; more precisely, if $(\lambda, L) \equiv(\lambda, \infty)$, $(-\infty, L)\left(\lambda, L\right.$ finite) then, respectively, $L^{\prime}=\infty$ or $\lambda^{\prime}=-\infty$. If $(\lambda, L) \equiv(-\infty$, $\infty)$, then $\left(\lambda^{\prime}, L^{\prime}\right) \equiv(-\infty, \infty)[4]$. 
By a "non-reducible" or "true" interval of orthogonality we mean [4] the interval determined by the limits of the least and greatest roots of $\phi_{n}(x)$ as $n$ increases indefinitely. Stieltjes [1] proved, for the interval $(0, \infty)$, to which we can always reduce the intervals $(\lambda, \infty),(-\infty, L)$ ( $\lambda$ or $L$ finite), that, if the largest root of $\phi_{n}(x)$ approaches infinity with $n$, then an infinite sequence of such roots approach infinity. Since we know that the zeros of $\phi_{n}(x)$ separate those of $\Omega_{n}(x)$, the theorem is established for the simply infinite interval. We need thus consider only the case of $(\lambda, L) \equiv(-\infty, \infty)$. For this purpose we make use of the following results of Hamburger [3]: $\psi_{n}(x)$ is defined as a weight function of order $n$ relative to the positive definite series

if

$$
P\left(\frac{1}{x}\right) \equiv \sum_{n=0}^{\infty} \frac{\alpha_{n}}{x^{n+1}} \sim \int_{-\infty}^{\infty} \frac{d \psi(y)}{x-y}
$$

(a) $\psi_{n}(x)$ is a step function with exactly $n$ points of increase, $x_{n, i}$. Let the saltus at such a point equal $M_{n, i}(i=1,2, \cdots, n)$. Thus

$$
\begin{array}{ll}
\psi_{n}(x)=0\left(-\infty \leqq x<x_{n, 1}\right), & \psi_{n}(x)=M_{n, 1}\left(x_{n, 1} \leqq x<x_{n, 2}\right), \\
\psi_{n}(x)=M_{n, 2}\left(x_{n, 2} \leqq x<x_{n, 3}\right), & \psi_{n}(x)=\alpha_{0}\left(x_{n, n} \leqq x \leqq+\infty\right) .
\end{array}
$$

(b) At least the first $(2 n-1)$ moments of the weight function $\psi_{n}(x)$ are identical with those of $\psi(x)$ :

$$
\int_{-\infty}^{\infty} x^{\nu} d \psi_{n}(x)=\alpha_{\nu} \quad(\nu=0,1,2, \cdots, 2 n-1) .
$$

From the theory of continued fractions [1] we know that the zeros of $\phi_{n+1}(x)$ separate those of $\phi_{n}(x)$, i.e., if the zeros of $\phi_{n}(x)$, in order of magnitude, are denoted by $x_{n, 1}, x_{n, 2}, \cdots, x_{n, n}$ we have

$$
x_{n+1,1}<x_{n, 1}<x_{n+1,2}<x_{n, 2}<\cdots<x_{n, n-1}<x_{n+1, n}<x_{n, n}<x_{n+1, n+1} .
$$

This shows that the largest zero and the next largest zero of $\phi_{n}(x)$ increase with $n$. Since we are dealing with a true interval of orthogonality, we have, by hypothesis,

$$
x_{n, n} \rightarrow+\infty, x_{n, 1} \rightarrow-\infty \quad(n \rightarrow \infty) .
$$

Let us assume

$$
x_{n, n-1} \rightarrow L^{\prime}, \quad x_{n, 2} \rightarrow \quad\left(n \rightarrow \infty ; \lambda^{\prime}, \text { or } L^{\prime} \text {, or both, finite }\right) .
$$

We then prove that assumption (B) contradicts the hypothesis (A).

Consider the relations [2] 


$$
\begin{aligned}
\frac{\Omega_{n}(x)}{\phi_{n}(x)}=\sum_{i=1}^{n} \frac{M_{n, i}}{x-x_{n, i}} & \\
& \left(\sum_{i=1}^{n} M_{n, i} x_{n, i}^{\prime}=\alpha_{\nu} ; \nu=0,1,2, \cdots, 2 n-1 ; M_{n, i}>0\right) .
\end{aligned}
$$

In particular

$$
\sum_{i=1}^{n} M_{n, i} x_{n, i}^{2}=\alpha_{2}=\int_{-\infty}^{\infty} x^{2} d \psi(x)>0
$$

It follows that

$$
M_{n, n} x_{n, n}^{2}<\alpha_{2}, M_{n, n} \rightarrow 0(n \rightarrow \infty) \text { (by (A)). }
$$

In the relations which define $\psi_{n}(x)$, let us take for $M_{n, i}$ and $x_{n, i}$ the same quantities as in (15). Then, as was shown by Hamburger [3], (b) is satisfied. Furthermore, there is a sub-sequence $\left\{\psi_{n \nu}(x)\right\}(\nu=1,2, \cdots)$ which, for $\nu \rightarrow \infty$, approaches $\psi(x)$ as a limit at all its points of continuity. For brevity we shall write $\psi_{\nu}(x)$ in place of $\psi_{n_{\nu}}(x)$. Then

$$
\psi_{\nu}(x)=\sum_{i=1}^{\nu} M_{\nu, i}=\alpha_{0} \quad\left(x_{\nu, \nu} \leqq x \leqq+\infty\right) .
$$

Take $x=L^{\prime \prime}$, any point of continuity of $\psi(x)$, such that $x_{\nu, \nu-1}<L^{\prime}<L^{\prime \prime}<x_{\nu, \nu}$. Then

and

$$
\psi_{\nu}\left(L^{\prime \prime}\right)=\sum_{i=1}^{\nu} M_{\nu, i}=\alpha_{0}-M_{\nu, \nu}
$$

$$
\lim _{\nu=\infty} \psi_{\nu}\left(L^{\prime \prime}\right)=\psi\left(L^{\prime \prime}\right)=\alpha_{0}-\lim _{\nu=\infty} M_{\nu, \nu}=\alpha_{0} .
$$

Therefore, since $\psi(\infty)=\alpha_{0}, \psi(x)$ is constant in the interval $\left(L^{\prime}, \infty\right)$ and all integrals involving $d \psi(x)$ would have $L^{\prime}$ for the upper limit of integration. Hence, $x_{\nu, \nu}<L^{\prime}$, for all $\nu$, in contradiction to (A).*

In similar manner, using $M_{\nu, 1}$ instead of $M_{\nu, \nu}$, we can show that the assumption $\lim _{n=\infty} x_{n, 2}=\lambda^{\prime}, \lambda^{\prime}$ finite, involves a contradiction $(\psi(x)=0$ in $\left.\left(-\infty, \lambda^{\prime}\right)\right)$. This proves our theorem for, if $\lim x_{n, n-1}=\lim x_{n, n}=\infty$ and $\lim x_{n, 2}=\lim x_{n, 1}=-\infty(n \rightarrow \infty)$, then, due to separation, the greatest and the least zeros of $\Omega_{n}(x)$ approach $+\infty$ and $-\infty$, respectively. Henceforth we shall, in general, denote by $(a, b)$ the true interval of orthogonality, finite or infinite, for the set $\left\{\phi_{n}(x)\right\}$.

* It is known that, if

$$
\Gamma_{a}^{b} \phi_{m}(x) \phi_{n}(x) d \psi(x)=0, m \neq n \quad(m, n \neq 0,1,2, \cdots)
$$

then all zeros of $\phi_{n}(x)(n=1,2, \cdots)$ are real, distinct and between $a$ and $b$. 
2. We now turn to the corresponding continued fraction $W(z)$ as given in (2) and its convergents $U_{n}(z) / V_{n}(z)$. Hereafter we assume, with Stieltjes $[1]$, that $b_{i}>0(i=1,2, \cdots)$. Then the corresponding Stieltjes integral is of the form

$$
\int_{0}^{\infty} \frac{d \psi(u)}{z-u}=\sum_{i=0}^{\infty} \frac{\alpha_{i}}{z^{i+1}} \sim W(z) \quad\left(\alpha_{i}=\int_{0}^{\infty} x^{i} d \psi(x)\right)
$$

$((0, \infty)$ perhaps being reducible to $(a, b)$ with $a>0[1,2])$. Since we get the continued fraction (1) by contraction from (2), $U_{2 n}(z) \equiv \Omega_{n}(z), V_{2 n}(z) \equiv \phi_{n}(z)$ $(n=0,1,2, \cdots)$, we shall restrict ourselves to the odd convergents $U_{2 n-1}(z)$ $/ V_{2 n-1}(z)(n \geqq 1)$. From the difference relations

$$
\begin{aligned}
& U_{2 n-1}(z)=z U_{2 n-2}(z)-b_{2 n-1} U_{2 n-3}(z), \\
& U_{2 n-2}(z)=U_{2 n-3}(z)-b_{2 n-2} U_{2 n-4}(z) \quad\left(n \geqq 2, U_{0} \equiv 0\right),
\end{aligned}
$$

with similar expressions for $V_{2 n-1}(z), V_{2 n-2}(z)$, we derive easily

$$
\begin{array}{r}
U_{2 n-1}(z)=\left[z-\left(b_{2 n-1}+b_{2 n-2}\right)\right] J_{2 n-3}(z)-b_{2 n-2} b_{2 n-3} U_{2 n-5}(z) \\
{\left[n>2, U_{1}(z)=b_{1} ; U_{3}(z)=b_{1}\left(z-b_{3}\right)\right],} \\
V_{2 n-1}(z)=\left[z-\left(b_{2 n-1}+b_{2 n-2}\right)\right] V_{2 n-3}(z)-b_{2 n-2} b_{2 n-3} V_{2 n-5}(z) \\
{\left[n>2 ; V_{1}(z)=z ; V_{3}(z)=z\left(z-b_{2}-b_{3}\right)\right] .}
\end{array}
$$

Since $U_{2 n-1}(z)$ and $V_{2 n-1}(z)$ are polynomials of degree $(n-1)$ and $n$, respectively, with $V_{2 n-1}(0)=0$, let

$$
U_{2 n-1}(z)=Q_{n-1}(z), \quad V_{2 n-1}(z)=z S_{n-1}(z) \quad(n \geqq 1)
$$

$\left(Q_{n-1}(z), S_{n-1}(z)\right.$ are polynomials of degree $\left.n-1\right)$. The difference equations (17) and (18) then lead to the following continued fractions of type (1):

$$
K^{\prime \prime}(z) \equiv \frac{b_{2} \mid}{\mid z-b_{3}}-\frac{b_{3} b_{4} \mid}{\mid z-b_{4}-b_{5}}-\frac{b_{5} b_{6} \mid}{\mid z-b_{6}-b_{7}}-\cdots,
$$

with convergents $P_{n}(z) / Q_{n}(z)$,

$$
K^{\prime \prime \prime}(z) \equiv \frac{b_{1} b_{2} \mid}{\mid z-b_{2}-b_{3}}-\frac{b_{3} b_{4} \mid}{\mid z-b_{4}-b_{5}}-\frac{b_{5} b_{6} \mid}{\mid z-b_{6}-b_{7}}-\cdots,
$$

with convergents $R_{n}(z) / T_{n}(z)$.

Consider first $K^{\prime \prime \prime}(z)$. Compare $S_{n}(z)$ as computed from (18) and (19) with $T_{n}(z)$ as computed from (21) (making $S_{0}(z)=T_{0}(z)=1$ ). We see that

$$
S_{n}(z)=T_{n}(z) \quad(n=1,2, \cdots) .
$$


Moreover, all the $b_{i}(i=1,2, \cdots)$ being positive, $K^{\prime \prime \prime}(z)$ may be formally associated with a positive definite series, $P_{1}(1 / z) \sum_{i=0}^{\infty} \beta_{i} / z^{i+1}$ (in the sense of (3)) the coefficients, $\beta_{i}$, of which may be computed through known formulas [2], and also with a Stieltjes integral $\int_{0}^{\infty}(1 /(z-u)) d \psi_{2}(u)$ of type (16), so that

$$
\int_{0}^{\infty} \frac{d \psi_{2}(u)}{z-u} \equiv \sum_{i=0}^{\infty} \frac{\beta_{i}}{z^{i+1}} \sim K^{\prime \prime \prime}(z) \quad\left(\beta_{i}=\int_{0}^{\infty} x^{i} d \psi_{2}(x)\right) .
$$

Hence, by the fundamental property of association (see (4), (5)),

$$
\int_{0}^{\infty} T_{m}(x) T_{n}(x) d \psi_{2}(x)=0 \quad(m \neq n ; m, n=0,1,2, \cdots) .
$$

Let

$$
P\left(\frac{1}{z}\right)=\sum_{i=0}^{\infty} \frac{\alpha_{i}}{z^{i+1}}
$$

be the series associated with $K(z)$, as given in (1), which, as was stated, is obtained by contraction from $W(z)$ in (2). Hence, since [5]

$$
\lambda_{n}=b_{2 n-2} b_{2 n-1}, c_{n}=b_{2 n+1}+b_{2 n}(n \geqq 2) ; \quad \lambda_{1}=b_{1}, c_{1}=b_{2},
$$

$K(z)$ can be re-written as follows:

$$
K(z) \equiv \frac{b_{1} \mid}{\mid z-b_{2}}-\frac{b_{2} b_{3} \mid}{\mid z-b_{3}-b_{4}}-\frac{b_{4} b_{5} \mid}{\mid z-b_{5}-b_{6}}-\cdots .
$$

We have then for the $\alpha_{i}[2]$

$$
\alpha_{0}=\lambda_{1}=b_{1}, \alpha_{1}=\lambda_{1} c_{1}=b_{1} b_{2}, \alpha_{2}=\lambda_{1}\left(\lambda_{2}+c_{1}^{2}\right)=b_{1} b_{2}\left(b_{2}+b_{3}\right), \cdots .
$$

In a similar manner we can express the $\beta_{i}$ related to the continued fraction $K^{\prime \prime \prime}(z)$. A simple comparison of the values of $\alpha_{i}$ and $\beta_{i}$ thus obtained gives the relation

$$
\beta_{i}=\alpha_{i+1} \quad(i=0,1,2, \cdots) .
$$

But, according to Stieltjes,

$$
\begin{aligned}
K(z)=\lim _{n=\infty} \frac{\Omega_{n}(z)}{\phi_{n}(z)}=\lim _{n=\infty} \frac{A_{2 n}(z)}{B_{2 n}(z)}=\int_{0}^{\infty} \frac{d \psi(u)}{z-u}, z \operatorname{not} \text { in }(0, \infty), \\
\alpha_{i}=\int_{0}^{\infty} x^{i} d \psi(x) \quad(i=0,1,2, \cdots),
\end{aligned}
$$

and (25) shows that a solution of the $\left[\beta_{i}\right]_{0}^{\infty}$-moments problem is $\psi_{2}(x)$, determined by the relation

$$
d \psi_{2}(x)=x d \psi(x) .
$$


Combining (19) and (22) we get the following result:*

$$
\begin{aligned}
\int_{0}^{\infty} \phi_{m}(x) \phi_{n}(x) d \psi(x)=0 \text { implies } \int_{0}^{\infty} T_{m}(x) T_{n}(x) x d \psi(x)=0 \\
\\
\quad(m \neq n ; m, n=0,1,2, \cdots) .
\end{aligned}
$$

We now turn to $K^{\prime \prime}(z)$, as given in (20). The very form of (20) shows, by reasoning similar to that employed above, that

$$
\int_{0}^{\infty} Q_{m}(x) Q_{n}(x) d \psi_{3}(x)=0, m \neq n \quad(m, n=0,1,2, \cdots) .
$$

Comparison of $K^{\prime}(x)$ and $K^{\prime \prime}(x)$ (see (7), (20), (24) combined with what we know about the distribution of the roots of $\Omega_{n}(x)$ ) shows that

$$
\int_{0}^{\infty} \Omega_{m}(x) \Omega_{n}(x) d \psi_{1}(x)=0, m \neq n \quad(m, n=0,1,2, \cdots) .
$$

Furthermore, if we compare the coefficients of the power series of type (23), associated with $K^{\prime}(z)$ and $K^{\prime \prime}(z)$ respectively, we find again, as above, that we may take in (26) and (26a)

$$
x d \psi_{3}(x)=d \psi_{1}(x),
$$

i.e. the relations (26) may be rewritten as

$$
\int_{0}^{\infty} Q_{m}(x) Q_{n}(x) \frac{1}{x} d \psi_{1}(x)=0, \quad m \neq n \quad(m, n=0,1,2, \cdots) .
$$

Let, further,

$$
K^{\mathrm{iv}}(z) \equiv \frac{b_{3} b_{4} \mid}{\mid z-b_{4}-b_{5}}-\frac{b_{5} b_{6} \mid}{\mid z-b_{6}-b_{7}}-\cdots .
$$

Then, following the method used in Lemma 4, it can be shown that if $K^{\text {iv }}(z)$ is completely convergent, so is $K^{\prime \prime}(z)$, and that the complete convergence of $K(z)$ implies that of $K^{\prime \prime \prime}(z)$, which in turn implies the complete convergence of $K^{\text {iv }}(z)$. Hence, the complete convergence of $K(z)$ implies that of $K^{\prime \prime}(z)$. The discussion of the intervals of orthogonality is similar to that of the previous case. We may, then, combine our results into the general

Theorem IV. A Stieltjes continued fraction of type (2)

$$
W(z)=\frac{b_{1} \mid}{\mid z}-\frac{b_{2} \mid}{\mid 1}-\frac{b_{3} \mid}{\mid z}-\frac{b_{4} \mid}{\mid 1}-\cdots \quad\left(b_{i}>0 ; i=1,2,3, \cdots\right)
$$

* This result was first obtained, in an entirely different manner, by J. Shohat [6]. 
with the convergents $U_{i}(z) / V_{i}(z)(i=0,1,2, \cdots)$ gives rise to four sets of orthogonal polynomials of degree $0,1,2, \cdots$ :

(a) $\quad \int_{0}^{\infty} V_{2 m}(x) V_{2 n}(x) d \psi(x)=0$,

(b) $\quad \int_{0}^{\infty} U_{2 m}(x) U_{2 n}(x) d \psi_{1}(x)=0$,

(c) $\quad \int_{0}^{\infty} S_{m}(x) S_{n}(x) x d \psi(x)=0 \quad\left(S_{n}(x) \equiv \frac{V_{2 n+1}(x)}{x}, n \geqq 1 ; S_{0}(x)=1\right)$,

(d) $\quad \int_{0}^{\infty} Q_{m}(x) Q_{n}(x) \frac{1}{x} d \psi_{1}(x)=0$

$$
\begin{array}{r}
\left(Q_{n}(x) \equiv U_{2 n+1}(x)\right) \\
(m \neq n ; m, n=0,1,2,3, \cdots) .
\end{array}
$$

If the moments problem related to the set $\left\{\phi_{n}(x)\right\} \equiv\left\{V_{2 n}(x)\right\}$ is determined then the moments problems related to the other three sets are also determined.

We notice that the main feature of our proof consisted in constructing continued fractions of the type $K(z)$ for which each of the sets of polynomials under discussion are the denominators of the successive convergents.

3. Differential equation for $\Omega_{n}(x)$. If $d \psi(x)=p(x) d x$, and $p(x)$ is of the form

$$
p(x)=e^{Q(x)} \prod_{i=1}^{\dot{1}}\left(x-a_{i}\right)^{A_{i}} \quad\left(A_{i}>-1 ; a_{i}=\text { const. } ; Q(x) \text { a polynomial }\right)
$$

then it has been shown by J. Shohat [7] that

$$
F^{\prime}(x)=T(x) F(x)+R(x)
$$

$$
\left(F(x)=\int_{a}^{b} \frac{p(y)}{x-y} d y\right)
$$

$\left(T(x)=p^{\prime}(x) / p(x), R(x)\right.$ a rational function), and that the corresponding orthogonal polynomials satisfy a homogeneous linear differential equation of second order

$$
\begin{aligned}
A_{n}(x) y_{n}^{\prime \prime}(x)+B_{n}(x) y_{n}^{\prime}(x)+C_{n}(x) y_{n}(x) & =0 \\
& \left(A_{n}(x), B_{n}(x), C_{n}(x) \text { polynomials }\right) .
\end{aligned}
$$

In particular, for the classical orthogonal polynomials, of Jacobi, Laguerre and Hermite, $p(x)$ is of type (27) and (29) assumes a very simple form. In fact $A_{n}(x), B_{n}(x)$ are polynomials independent of $n$, of degree $\leqq 2,1$, respectively, while $C_{n}$ is a constant depending on $n$ only. They are as follows: 


\begin{tabular}{|c|c|c|c|c|c|}
\hline & $p(x)$ & $\begin{array}{c}\text { interval of } \\
\text { orthogonality }\end{array}$ & $A_{\boldsymbol{n}}(x) \equiv A(x)$ & $B_{n}(x) \equiv B(x)$ & $C_{n}(x) \equiv C_{n}$ \\
\hline Jacobi $(J)$ & $\begin{array}{c}(x-a)^{\alpha-1}(b-x)^{\beta-1} \\
(\alpha, \beta>0)\end{array}$ & $(a, b)$ finite & $(x-a)(b-x)$ & $\alpha b-\beta a-(\alpha+\beta) x$ & $n(\alpha+\beta+n-1)$ \\
\hline Laguerre (L) & $\begin{array}{l}e^{-x} x^{\alpha-1} \\
(\alpha>0)\end{array}$ & $(0, \infty)$ & $x$ & $\alpha-x$ & $n$ \\
\hline Hermite $(\mathrm{H})$ & $e^{-x^{2}}$ & $(-\infty, \infty)$ & 1 & $-2 x$ & $2 n$ \\
\hline
\end{tabular}

Furthermore,

$$
p(x)=(1 / A(x)) \exp \int \frac{B(x)}{A(x)} d x .
$$

In order to derive a differential equation for $\Omega_{n}(x)$ we need one more relation from the theory of continued fractions [2]:

$$
\phi_{n}(x) F(x)=\Omega_{n}(x)+R_{n}(x) \quad\left(R_{n}(x),=\frac{\alpha^{\prime}}{x^{n+1}}+\frac{\alpha^{\prime \prime}}{x^{n+2}}+\cdots, \alpha^{\prime} \neq 0\right) .
$$

Moreover, [7], for $p(x)$ of thetype (27), $R_{n}(x) / p(x)$ is a second solution of the differential equation (29). From (32) we find

$$
R_{n}(x) / p(x)=\phi_{n}(x) F(x) / p(x)-\Omega_{n}(x) / p(x) .
$$

Substituting this expression into (29), we find the desired equation after a somewhat lengthy but simple computation:

$$
\begin{aligned}
A_{n}(x) \Omega_{n}^{\prime \prime}(x)+\left[B_{n}(x)-2 A_{n}(x) p^{\prime}(x) / p(x)\right] \Omega_{n}^{\prime}(x)+\left[C_{n}(x)-B_{n}(x) p^{\prime}(x) / p(x)\right. \\
\left.\quad-A_{n}(x) p^{\prime \prime}(x) / p(x)+2 A_{n}(x) p^{\prime}(x)^{2} / p(x)^{2}\right] \Omega_{n}(x) \\
=\left[A_{n}(x) R^{\prime}(x)-\left(A_{n}(x) p^{\prime}(x) / p(x)\right) R(x)+B_{n}(x) R(x)\right] \phi_{n}(x) \\
\quad+2 A_{n}(x) R(x) \phi_{n}^{\prime}(x) .
\end{aligned}
$$

(33) can be very greatly simplified for the classical polynomials (30). We show that here $R(x)$ has a very simple expression:

$$
R(x)=\left(A^{\prime \prime}(x)-B^{\prime}(x)+C_{1}\right) / A(x) \equiv k_{1} / A(x) \quad \text { (see (36) below). }
$$

Using the expression for $p(x)$ from (31), (33) becomes

$$
\begin{aligned}
& A(x) \Omega_{n}^{\prime \prime}(x)+ {\left[2 A^{\prime}(x)-B(x)\right] \Omega_{n}^{\prime}(x)+\left[A^{\prime \prime}(x)-B^{\prime}(x)+C_{n}\right] \Omega_{n}(x) } \\
&=[A(x) R(x)]^{\prime} \phi_{n}(x)+2 A(x) R(x) \phi_{n}^{\prime}(x) \quad(n=1,2, \cdots) .
\end{aligned}
$$

We now let $n=1$, and observe that $\Omega_{n}(x)$ is a polynomial of degree $n-1$, and that $A(x)$ and $B(x)$ do not depend on $n$. Making the leading coefficient of $\Omega_{n}(x)$ equal 1 we obtain 


$$
\left(A^{\prime \prime}(x)-B^{\prime}(x)+C_{1}\right)=[A(x) R(x)]^{\prime}\left(x-c_{1}\right)+2 A(x) R(x) .
$$

Let (see (30))

$$
A^{\prime \prime}(x)-B^{\prime}(x)+C_{n}=k_{n} \text { (constant independent of } x \text { ). }
$$

We have

$$
[A(x) R(x)]^{\prime}\left(x-c_{1}\right)+2 A(x) R(x)=k_{1},
$$

and this we treat as a differential equation in $A(x) R(x)$, which gives

$$
A(x) R(x)=\frac{D}{\left(x-c_{1}\right)^{2}}+k_{1} / 2 \quad(D=\text { const. }) .
$$

It is now easy to show that $D=0$. In fact, substituting (37) into (35), we get, for $n=2$, on the right side

$$
\begin{aligned}
& -2 D /\left(x-c_{1}\right)^{3} \phi_{2}(x)+2\left[D /\left(x-c_{1}\right)^{2}+k_{1} / 2\right] \phi_{2}^{\prime}(x) \\
& \quad=k_{1} \phi_{2}^{\prime}(x)-\left[2 D /\left(x-c_{1}\right)^{3}\right]\left[\phi_{2}(x)-\left(x-c_{1}\right) \phi_{2}^{\prime}(x)\right],
\end{aligned}
$$

and this can reduce to a polynomial (as in the left side of (35)), if and only if $D=0$.

We thus proved (34), which, substituted into (35), gives the following differential equation for $\Omega_{n}(x)$ in the classical cases:

$$
\begin{aligned}
A(x) \Omega_{n}^{\prime \prime}(x)+\left[2 A^{\prime}(x)-B(x)\right] \Omega_{n}^{\prime}(x)+k_{n} \Omega_{n}(x)=k_{1} \phi_{n}^{\prime}(x) \\
\left(k_{n}=A^{\prime \prime}(x)-B^{\prime}(x)+C_{n} ; n=1,2, \cdots\right) .
\end{aligned}
$$

The coefficients of the differential equation for the numerators are thus seen to be the coefficients of the adjoint of the differential equation (29) for the denominators.

We shall write equation (35) explicitly (see 30):

(39) $\mathrm{J}:(x-a)(b-x) \Omega_{n}^{\prime \prime}(x)+[2(a+b)-\alpha b-\beta a+(\alpha+\beta-4) x] \Omega_{n}^{\prime}(x)$

$$
+[(\alpha+\beta)(n+1)+n(n-1)-2] \Omega_{n}(x)=2(\alpha+\beta-1) \phi_{n}^{\prime}(x)[8] \text {. }
$$

(40) $\mathrm{L}: x \Omega_{n}^{\prime \prime}(x)-(\alpha-2-x) \Omega_{n}^{\prime}(x)+(n+1) \Omega_{n}(x)=2 \phi_{n}^{\prime}(x)$.

(41) $\mathrm{H}: \Omega_{n}^{\prime \prime}(x)+2 x \Omega_{n}^{\prime}(x)+2(n+1) \Omega_{n}(x)=4 \phi_{n}^{\prime}(x)$.

Making use of the simple expression of $R(x)$ as given in (34), we can find another expression for $F(x)$ from (28):

$$
F(x)=p(x)\left[\frac{k_{1}}{2} \int^{x} \frac{d x}{A(x) p(x)}+C\right] \quad(C=\text { const. }) .
$$

Remarks. (i) If we differentiate (38) twice and, where $y_{n} \equiv \phi_{n}(x)$, (29) once, eliminate $\phi_{n}{ }^{i}(x)(i=0,1,2,3)$, we obtain for $\Omega_{n}(x)$ a linear homogenous equation of the 4 th order. 
(ii) The general solution of (38) is

$$
\left.\Omega_{n}(x)+D_{1} p(x) \phi_{n}(x)+D_{2} R_{n}(x) \quad \text { ( } D_{1,2}=\text { const. }\right)
$$

for [7] $p(x) \phi_{n}(x)$ and $R_{n}(x)$ are solutions of

$$
A(x) y_{n}^{\prime \prime}(x)+\left[2 A^{\prime}(x)-B(x)\right] y_{n}^{\prime}(x)+\left[A^{\prime \prime}(x)-B^{\prime}(x)+C_{n}\right] y_{n}(x)=0 .
$$

4. The differential equations for $\left\{\Omega_{n}(x)\right\}$ of Laguerre and Hermite cases as limiting cases of that for the $\left\{\Omega_{n}(x)\right\}$ of the Jacobi case. Following a method indicated by P. Appell and J. Kampé de Fériet [9], we write the differential equations for the $\left\{\Omega_{n}(x)\right\}$ for the Jacobi case in the interval $(0,1)$ and the characteristic function $x^{\alpha-1}(1-x)^{\beta-1}$ or $\Omega_{n}\left[x ; 0,1 ; x^{\alpha-1}(1-x)^{\beta-1}\right]$ :

$$
\begin{gathered}
x(1-x) \Omega_{n}^{\prime \prime}(x)+[2-\alpha+(\alpha+\beta-4) x] \Omega_{n}^{\prime}(x) \\
+[(\alpha+\beta)(n+1)+n(n-1)-2] \Omega_{n}(x) \\
=2(\alpha+\beta-1) n \phi_{n-1}\left[x ; 0,1 ; x^{\alpha}(1-x)^{\beta}\right]
\end{gathered}
$$

since in the classical cases generally $\phi_{n}^{\prime}[x ; a, b ; p]=n \phi_{n-1}^{\prime}[x ; a, b ; A p]$ where $A \equiv A(x)$ is the coefficient of $\phi_{n}^{\prime \prime}(x)$ in (29). In (43) let $\beta-1=s, x=x_{1} / s$, divide by $s^{1 / 2} s^{(1-\alpha / 2)}(\alpha+s-3)$ and then let $s \rightarrow \infty$. We thus obtain

$$
x_{1} \Omega_{n}^{\prime \prime}\left(x_{1}\right)+\left(2-\alpha-x_{1}\right) \Omega_{n}^{\prime}\left(x_{1}\right)+(n+1) \Omega_{n}\left(x_{1}\right)=2 \phi_{n}^{\prime}\left(x_{1}\right)
$$

which is the differential equation (40) for the $\Omega_{n}\left(x_{1}\right)$ in the Laguerre case.

Again, write the differential equation for $\Omega_{n}\left[x ;-1,1 ;(1+x)^{\alpha-1}(1-x)^{\beta-1}\right]$

$$
\begin{aligned}
& \left(1-x^{2}\right) \Omega_{n}^{\prime \prime}(x)+[-\alpha+\beta+(\alpha+\beta-4) x] \Omega_{n}^{\prime}(x) \\
& \quad+[(\alpha+\beta)(n+1)+n(n-1)-2] \Omega_{n}(x)=2(\alpha+\beta-1) \phi_{n}^{\prime}(x) ;
\end{aligned}
$$

let $\alpha-1=\beta-1=s, x=x_{1} s^{-1 / 2}$, divide through by $s^{1 / 4}(s-1)$, and let $s \rightarrow \infty$ :

$$
\Omega_{n}^{\prime \prime}\left(x_{1}\right)+2 x_{1} \Omega_{n}^{\prime}\left(x_{1}\right)+2(n+1) \Omega_{n}\left(x_{1}\right)=4 \phi_{n}^{\prime}\left(x_{1}\right),
$$

which is the differential equation (41) for the $\Omega_{n}\left(x_{1}\right)$ in the Hermite case.

Hence, the numerators as well as the denominators of the associated continued fractions of Laguerre and Hermite cases may be considered as limiting cases of those of the Jacobi case.

5. Relations between $\left\{\phi_{n}(x)\right\}$ and $\left\{\Omega_{n}(x)\right\}$. (i) It is evident, from what has been said before, that we may write the associated continued fraction $K^{\prime}(x)$, having $\left\{\Omega_{n}(x)\right\}$ as denominators of its convergents, directly from the given $K(x)$. Also, given the corresponding continued fraction $W(x)$, we may, from (20) and (21), write the continued fractions of type $K(x)$ where $U_{2 n}(x)$, $U_{2 n+1}(x),(1 / x) V_{2 n+1}(x)$ will play the same rôle as $V_{2 n}(x)$. Let 


$$
\begin{aligned}
& \phi_{n}(x) \equiv x^{n}-S_{n} x^{n-1}+\cdots ; \\
& \Omega_{n}(x) \equiv x^{n-1}-\sigma_{n-1} x^{n-2}+\cdots \quad(n=1,2, \cdots) .
\end{aligned}
$$

We know [5] that $S_{n}=\sum_{i=1}^{n} c_{i}$ and since $K^{\prime}(x)$ is obtained by raising the indices of the $\lambda_{i}$ 's and $c_{i}$ 's by 1 , it follows directly that

$$
\sigma_{n}=S_{n+1}+c_{1} \quad(n=1,2, \cdots) .
$$

Let $a_{n}, \rho_{n}(n=0,1, \cdots)$ represent the "normalizing factors" for the sets $\left\{\phi_{n}(x)\right\},\left\{\Omega_{n}(x)\right\}$ respectively, i.e.

$$
\begin{gathered}
\int_{-\infty}^{\infty} \phi_{m}(x) \phi_{n}(x) d \psi(x)=\int_{-\infty}^{\infty} \omega_{m}(x) \omega_{n}(x) d \psi_{1}(x)=\delta_{m, n}(m, n=0,1,2, \cdots), \\
\phi_{n}(x) \equiv a_{n} \phi_{n}(x), \quad \omega_{n}(x) \equiv \rho_{n} \Omega_{n+1}(x) .
\end{gathered}
$$

We know [5] that

$$
a_{n}^{2}=\left(\lambda_{1} \lambda_{2} \cdots \lambda_{n+1}\right)^{-1} \quad(n=0,1,2, \cdots) .
$$

It follows then, in the same manner as for (44), that

$$
\rho_{n}=\lambda_{1}^{1 / 2} a_{n+1} \quad(n=0,1, \cdots)
$$

if we choose the first partial numerator in $K^{\prime}(x)$ equal to $\lambda_{2}$, which can be done without any loss of generality.

(ii) Relations between the moments of the distributions $d \psi(x)$ and $d \psi_{1}(x)$. Introducing proper constant factors, we can choose $\psi(x), \psi_{1}(x)$ in (3), (13), so that $\alpha_{0}=\int_{-\infty}^{\infty} d \psi(x)=1 ; \beta_{0}=\int_{-\infty}^{\infty} d \psi_{1}(x)=\lambda_{2}$. The "associations"

$$
\begin{array}{ll}
F(x) \equiv P(1 / x)=\sum_{i=0}^{\infty} \frac{\alpha_{i}}{x^{i+1}} \sim K(x) & \left(\alpha_{i}=\int_{-\infty}^{\infty} x^{i} d \psi(x), \alpha_{0}=1\right), \\
F_{1}(x) \equiv P_{1}(1 / x)=\sum_{i=0}^{\infty} \frac{\beta_{i}}{x^{i+1}} \sim K^{\prime}(x) & \left(\beta_{i}=\int_{-\infty}^{\infty} x^{i} d \psi_{1}(x), \beta_{0}=\lambda_{2}\right)
\end{array}
$$

lead to the formal relation $F(x)=\left(x-c_{1}-F_{1}(x)^{-1}\right.$ from which we have the following relations between the $\alpha_{i}$ and $\beta_{i}$ :

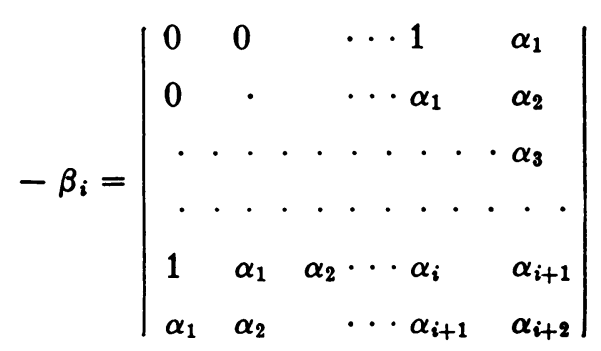


(iii) The symmetric case. Let the $\left\{\phi_{n}(x)\right\}$ be a set of "symmetric" orthogonal polynomials, i.e.

$$
S_{n}=c_{n}=0, \quad \phi_{n}(x) \equiv(-1)^{n} \phi_{n}(-x) \quad(n=1,2, \cdots) .
$$

Then from the similarity of the difference equations satisfied by $\left\{\phi_{n}(x)\right\}$ and $\left\{\Omega_{n}(x)\right\}$ (see (8), (9)), we conclude that the $\left\{\Omega_{n}(x)\right\}$ also form a symmetric set. However, as may be surmised from the fact that the set $\left\{\phi_{n}(x)\right\}$ involves one more essential constant $\left(c_{1}\right)$ than does the set $\left\{\Omega_{n}(x)\right\}$, the symmetry of the set $\left\{\phi_{n}(x)\right\}$ is not necessary for that of the set $\left\{\Omega_{n}(x)\right\}$. In fact, if we take the Jacobi case in the interval $(-1,1)$, then, from the general formula [10]

$$
\begin{array}{r}
c_{n}=(\alpha-\beta)(\alpha+\beta-2) /[(\alpha+\beta+2 n-2)(\alpha+\beta+2 n-4)] \\
\left(n>1 ; c_{1}=(\alpha-\beta) /(\alpha+\beta)\right),
\end{array}
$$

we see that, if $\alpha+\beta=2, \alpha \neq \beta$, then $c_{n}=0(n=2,3, \cdots)$, but $c_{1} \neq 0$. Thus, in this case, the set $\left\{\Omega_{n}(x)\right\}$ is symmetric while the set $\left\{\phi_{n}(x)\right\}$ is not.

6. Some particular classical cases. In this section we shall study some particular classical cases in which we obtain an explicit expression for $p_{1}(x)$, the characteristic function for the set $\left\{\Omega_{n}(x)\right\}$, also some simple relations between $\left\{\phi_{n}(x)\right\}$ and $\left\{\Omega_{n}(x)\right\}$.

(i) The sets $\left\{\phi_{n}(x)\right\}$ and $\left\{\Omega_{n}(x)\right\}$ are identical (disregarding constant factors), i.e.

$$
\phi_{n}(x) \equiv \Omega_{n+1}(x) \quad(n=0,1,2, \cdots) .
$$

For the sake of simplicity we consider the case of symmetric $\left\{\phi_{n}(x)\right\}$. Our hypothesis leads to

$$
F(x)=D_{1} /\left(x-D_{2} F(x)\right) \quad\left(D_{1,2}=\text { const. }\right) .
$$

Solving this equation for $F(x)$ and choosing a proper sign for the radical and proper value for the constants $D_{1,2}$, we obtain

$$
F(x)=\pi /\left(x+\left(x^{2}-1\right)^{1 / 2}\right)=\int_{-1}^{1} \frac{\left(1-y^{2}\right)^{1 / 2}}{x-y} d y[10] .
$$

* The formula

$$
F(x)=\int_{-\infty}^{\infty} \frac{p(y) d y}{x-y}
$$

shows that for $|x| \rightarrow \infty, F(x) \rightarrow 0$. On the other hand, in the continued fraction

$$
\frac{D_{1} \mid}{\mid x}-\frac{D_{2} \mid}{\mid x}-\cdots \text {, }
$$

the $\phi_{n}(x)$ do not depend on $D_{1}$, the $\Omega_{n}(x)$ do not depend on $D_{2}$ and contain $D_{1}$ as a factor. Hence having $D_{3}$ we can choose $D_{1}$ so as to bave $D_{1} D_{2}=1$. 
Hence

$$
(a, b)=(-1,1) ; \quad \psi(x)=\int_{-1}^{x}\left(1-x^{2}\right)^{1 / 2} d x, \quad p(x)=\left(1-x^{2}\right)^{1 / 2},
$$

and the above is the only case in which, under the given conditions, (45) is satisfied. The explicit expression for $\Omega_{n}(x)$ is $(\sin n \theta) / \sin \theta(x=\cos \theta)$. $\dagger$

(ii) $\phi_{n}^{\prime}(x)=\Omega_{n}(x)(n=1,2, \cdots)$ (within a constant factor). The differential equation (38) for the $\Omega_{n}(x)$ where we substitute now $\phi_{n}^{\prime}(x) / n=\Omega_{n}(x)$ gives

$$
A(x) \Omega_{n}^{\prime \prime}(x)+\left(2 A^{\prime}(x)-B(x)\right) \Omega_{n}^{\prime}(x)+\left(C_{n}-C_{1}\right) \Omega_{n}(x)=0 .
$$

Applying (31) to (46), we obtain

$$
p_{1}(x)=1 / p(x) .
$$

But we know that the characteristic function for $\phi_{n}^{\prime}(x)$ is $A(x) p(x)$.

Hence,

$$
p_{1}(x)=1 / p(x)=A(x) p(x) ; \quad p(x)=A(x)^{-1 / 2} .
$$

In view of (30), the only case when (47) holds is the Jacobi case for $\alpha=\beta=1 / 2$ where the $\phi_{n}(x)$ are the so-called "trigonometric polynomials" $\cos n \operatorname{arc} \cos x$ and

$$
\Omega_{n}(x)=\frac{\sin n \theta}{\sin \theta} \quad(x=\cos \theta) .
$$

The characteristic [10] function for the $\left\{\phi_{n}(x)\right\}$ is, in the interval $(-1,1)$, $\left(1-x^{2}\right)^{-1 / 2}$ and the characteristic function for the $\left\{\Omega_{n}(x)\right\}$ is, by (47), $\left(1-x^{2}\right)^{1 / 2}$.

It is interesting to note that the $\Omega_{n}(x)$ so obtained are the same as in (i), for $p_{1}(x)$ obtained from (47) is identical with that found in (i).

(iii) $\Omega_{n}(x)$ of Jacobi case, in the interval $(-1,1)$ with $\alpha+\beta=1$, i.e. with $p(x)=(1+x)^{\alpha-1}(1-x)^{-\alpha}(1>\alpha>0)$. The differential equation (39) for $\Omega_{n}(x)$ of the Jacobi case in the interval $(-1,1)$, with arbitrary $\alpha, \beta>0$, is

$$
\begin{aligned}
& \left(1-x^{2}\right) \Omega_{n}^{\prime \prime}(x)+[-\alpha+\beta+(\alpha+\beta-4) x] \Omega_{n}^{\prime}(x) \\
& \quad+[(\alpha+\beta)(n+1)+n(n-1)-2] \Omega_{n}(x)=2(\alpha+\beta-1) \phi_{n}^{\prime}(x) .
\end{aligned}
$$

$\dagger$ Here, disregarding constant factors,

$$
\frac{\Omega_{n}(\cos \theta)}{\phi_{n}(\cos \theta)}=\frac{\sin n \theta}{\sin (n+1) \theta}=\frac{\left(x+\left(x^{2}-1\right)^{1 / 2}\right)^{n}-\left(x-\left(x^{2}-1\right)^{1 / 2}\right)^{n}}{\left(x+\left(x^{2}-1\right)^{1 / 2}\right)^{n+1}-\left(x-\left(x^{2}-1\right)^{1 / 2}\right)^{n+1}} .
$$

If we take $|x|>1$ and let $n \rightarrow \infty$ we get in the limit $\left(x+\left(x^{2}-1\right)^{1 / 2}\right)^{-1}=F(x) / \pi$ in accordance with Markoff's theorem. 
The case $\alpha+\beta=1$ deserves special attention, for then (48) becomes a homogenous differential equation of the same type as that for $\phi_{n}(x)$ :

$$
\begin{array}{r}
\left(1-x^{2}\right) \Omega_{n}^{\prime \prime}(x)+[1-2 \alpha-3 x] \Omega_{n}^{\prime}(x)+(n+1)(n-1) \Omega_{n}(x)=0 \\
(n=1,2, \cdots) .
\end{array}
$$

Comparing (49) with (30) (where $n, \alpha, \beta$ are replaced by $n-1,2-\alpha, \alpha+1$ respectively) we conclude that the $\Omega_{n}(x)$ are identical with the $\phi_{n}(x)$ corresponding to $(1+x)^{1-\alpha}(1-x)^{\alpha}=1 / p(x)$. For the interval $(0,1)$, making use of Theorem IV, we thus obtain

THEOREM V. If in the continued fraction $W(x)$, given by (2), with the convergents $U_{i}(x) / V_{i}(x)(i=0,1, \cdots)$, the set $\left\{V_{2 n}(x)\right\}$ is orthogonal in $(0,1)$ with the characteristic function $x^{\alpha-1}(1-x)^{-\alpha}(1>\alpha>0)$, then the other three sets, $\left\{(1 / x) V_{2 n+1}(x)\right\},\left\{U_{2 n}(x)\right\},\left\{U_{2 n+1}(x)\right\}$, are orthogonal in $(0,1)$ with the characteristic functions $x^{\alpha}(1-x)^{-\alpha}, x^{1-\alpha}(1-x)^{\alpha}, x^{-\alpha}(1-x)^{\alpha}$, respectively.

The trigonometric polynomials considered above in (ii) are evidently a special case $\alpha=\beta=1 / 2$.

(iv) Using the difference equations ((17), (18)) and the fact that $K^{\prime \prime \prime}(x)$ has, for denominators of its convergents, polynomials orthogonal with respect to the distribution $x d \psi(x)\left(b_{1}=1\right)$, we obtain

$$
V_{2 n}(x d \psi(x))+U_{2 n}(x d \psi(x))=U_{2 n+1}(d \psi(x)) \quad\left(b_{1}=1 ; n=1,2, \cdots\right) .
$$

In fact, $T_{i}(x)+R_{i}(x)=Q_{i}(x)(i=0,1)$, and $T_{n}(x)$ and $R_{n}(x)$ satisfy the same difference equation, except for initial conditions, as $Q(x)$; then

$$
R_{n}(x)+T_{n}(x)=Q_{n}(x) \quad(n=1,2, \cdots) .
$$

7. Expansion of $\left\{\Omega_{n}(x)\right\}$ in terms of $\left\{\phi_{n}(x)\right\}$. Our starting point is the integral representation of $\Omega_{n}(x)$ [2],

$$
\Omega_{n}(x)=\int_{a}^{b} \frac{\phi_{n}(x)-\phi_{n}(y)}{x-y} d \psi(y) .
$$

We find easily, writing

$$
\begin{aligned}
\frac{\phi_{n}(x)-\phi_{n}(\ddot{y})}{x-y} & =L_{n, n-1}(y) \phi_{n-1}(x)+L_{n, n-2}(y) \phi_{n-2}(x)+\cdots, \\
L_{n, n-1}(y) & =1, \\
L_{n, n-2}(y) & =y-c_{n}, \\
L_{n, n-3}(y) & =\left(y-c_{n-1}\right) L_{n, n-2}(y)-\lambda_{n} L_{n, n-1}(y), \\
L_{n, n-4}(y) & =\left(y-c_{n-2}\right) L_{n, n-3}(y)-\lambda_{n-1} L_{n, n-2}(y),
\end{aligned}
$$


We may thus exhibit $L_{n, n-i-1}(y)$ as the denominators of the $i$ th convergent of the continued fraction

$$
\frac{1}{\mid y-c_{n}}-\frac{\lambda_{n} \mid}{\mid y-c_{n-1}}-\frac{\lambda_{n-1} \mid}{\mid y-c_{n-2}}-\cdots-\frac{\lambda_{3} \mid}{\mid y-c_{2}},
$$

whose relation to $K(y)$ is obvious. These denominators may also be expressed as determinants [2]. We have

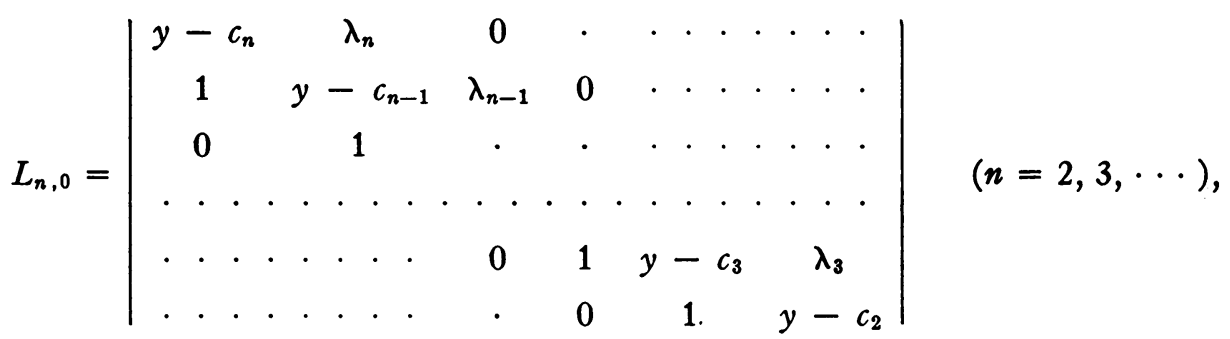

and $L_{n, n-i}(y)$ is formed from $L_{n, 0}(y)$ by dropping the $n-i$ last rows and columns.

Our expansion is then

$$
\begin{aligned}
\Omega_{n}(x) & =\sum_{i=1}^{n} A_{n, n-i} \phi_{n-i}(x), \\
A_{n, n-i} & =\int_{a}^{b} \Omega_{n}(x) \phi_{n-i}(x) d \psi(x)=\int_{a}^{b} L_{n, n-i}(x) d \psi(x) .
\end{aligned}
$$

Another method for obtaining the expansion under consideration is furnished by the differential equation (38). This method, first applied by Christoffel [8] to the Legendre case, may be epitomized as follows. Substitute in (38)

$$
\begin{aligned}
\phi_{n}^{\prime}(x) & =\sum_{i=1}^{n} g_{n, n-i} \phi_{n-i}(x), \\
\Omega_{n}(x) & =\sum_{i=1}^{n} A_{n, n-i} \phi_{n-i}(x) \quad\left(g_{n, n-i}, A_{n, n-i}=\text { const. }\right),
\end{aligned}
$$

and equate the coefficients of $\phi_{n-i}(x)$ (for an expansion of a polynomial in terms of $\phi_{i}(x)$ is necessarily unique). This leads to linear relations involving the $g_{n, n-i}$ and the $A_{n, n-i}$. These become especially simple in the cases which follow.

(i) Hermite case: $p(x)=e^{-x^{2}}$. This is the simplest case, since $\phi_{n}^{\prime}(x) \equiv$ $n \phi_{n-1}(x)$, i.e. $g_{n, n-1}=n, g_{n, n-2}=g_{n, n-3}=\cdots=g_{n, 0}=0$. Carrying out the indicated computation, we obtain 


$$
\begin{aligned}
\Omega_{n}(x)= & \sum_{i=0} A_{n, n-(2 m+1)} \phi_{n-(2 m+1)}(x), \\
\text { (54) } A_{n, n-(2 m+1)}= & {\left[(-1)^{m} / 2^{m}\right](n-m-1)(n-m-2) } \\
& -\cdots(n-2 m) \quad\left(m=1,2, \cdots,\left[\frac{n-1}{2}\right]\right) .
\end{aligned}
$$

(ii) Laguerre case: $p(x)=e^{-x}$. If we write out the explicit expression of Laguerre polynomials

$$
\phi_{n}(x)=x^{n}-n^{2} x^{n-1}+\frac{n^{2}(n-2)^{2}}{2 !} x^{n-2}+\cdots \quad(n=0,1, \cdots),
$$

we readily obtain the coefficients in the expansion (52) by direct computation:

$$
g_{n, n-i}=(-1)^{i+1} n ! /(n-i) ! \quad(n=1,2, \cdots ; i=1,2, \cdots, n) .
$$

Using this result we proceed as in the previous case:

$$
\Omega_{n}(x)=\sum_{i=1}^{n} A_{n, n-i} \phi_{n-i}(x)
$$

$$
\begin{aligned}
& A_{n, n-1}=1, \\
& A_{n, n-2}=-2(n-1) \\
& A_{n, n-3}=\frac{2(n-2)}{2 n-2}\left[\frac{n !}{(n-2) !}-(n-2) A_{n, n-2}\right], \\
& A_{n, n-4}=-\frac{2(n-3)}{2 n-3}\left[\frac{n !}{(n-3) !}+(n-3) A_{n, n-3}\right],
\end{aligned}
$$

8. Relation between $\psi(x)$ and $\psi_{1}(x)$. We assume that $K(z)$, hence $K^{\prime}(z)$, are related to determined moments problems whose solutions are $\psi(x)$ and $\psi_{1}(x)$ respectively. In order to establish a relation between these two functions we make use of the following formula (Perron [2], p. 372):

$$
\begin{array}{r}
\frac{\psi(x-0)+\psi(x+0)}{2}-\frac{\psi\left(x_{0}-0\right)+\psi\left(x_{0}+0\right)}{2}=\lim _{y=+0} R\left\{\frac{1}{\pi i} \int_{x_{0}+i y}^{x+i y} F(z) d z\right\} \\
\left(z=x+i y, a<x_{0}, x<b\right),
\end{array}
$$

where the path of integration is a straight line parallel to the $x$-axis. If we apply the same formula to $\psi_{1}(x)$ and express the result in terms of $\psi(x)$, using (10), we obtain, since 


$$
\lim _{y=+0} R\left\{\frac{1}{\pi i} \int_{x_{0}+i y}^{x+i y}\left(z-C_{1}\right) d z\right\}=0
$$

the fundamental relation

$$
\begin{aligned}
\frac{\psi_{1}(x-0)+\psi_{1}(x+0)}{2} & -\frac{\psi_{1}\left(x_{0}-0\right)+\psi_{1}\left(x_{0}+0\right)}{2} \\
& =\lim _{y=+0} R\left\{\frac{i}{\pi} \int_{x_{0}+i y}^{x+i y} \frac{1}{F(z)} d z\right\} \quad\left(a<x_{0}, x<b\right) .
\end{aligned}
$$

We may change, if necessary, the values of $\psi_{1}(x)$ at its points of discontinuity, so as to have on $(a, b) \psi_{1}(x) \equiv \frac{1}{2}\left(\psi_{1}(x-0)+\psi_{1}(x+0)\right)$. Then formula (56) becomes

$$
\psi_{1}(x)-\psi_{1}\left(x_{0}\right)=\lim _{y=+0} R\left\{\frac{i}{\pi} \int_{x_{0}+i y}^{x+i y} \frac{1}{F(z)} d z\right\} \quad\left(a<x_{0}, x<b\right) .
$$

If the explicit expression of the function

$$
G(z)=\int_{a}^{b} \frac{d \psi(u)}{z-u}
$$

which, for $z$ not in $(a, b)$, coincides with $F(z)$, is known and is such that $1 / G(z)$ is regular analytic inside $(a, b)$, with perhaps a finite number of singularities therein, we can obtain the explicit expression for $\psi_{1}(x)$, using (57), as follows: we choose $x$ and $x_{0}$ sufficiently close to $x$ so that $1 / G(x)$ has no singularities on the whole segment $\left(x_{0}, x\right)$. We have then, by Cauchy's Theorem,

$\frac{i}{\pi} \int_{x}^{x_{0}} \frac{d x}{G(x)}+\frac{i}{\pi} \int_{0}^{y} \frac{d y}{G\left(x_{0}+i y\right)}+\frac{i}{\pi} \int_{x_{0}+i y}^{x+i y} \frac{d z}{G(z)}+\frac{i}{\pi} \int_{y}^{0} \frac{d y}{G(x+i v)}=0$.

Hence

$$
\begin{aligned}
R\left\{\frac{i}{\pi} \int_{x_{0}+i y}^{x+i y} \frac{d z}{G(z)}\right\}=R\left\{\frac{i}{\pi} \int_{x_{0}}^{x} \frac{d x}{G(x)}\right\} \\
-R\left\{\frac{i}{\pi} \int_{0}^{y} \frac{d y}{G\left(x_{0}+i y\right)}\right\}+R\left\{\frac{i}{\pi} \int_{0}^{y} \frac{d y}{G(x+i y)}\right\} .
\end{aligned}
$$

Now let $y \rightarrow+0$. The last two integrals will approach zero, for

$$
\left|\frac{i}{\pi} \int_{0}^{y} \frac{d y}{G(c+i y)}\right| \leqq \frac{1}{\pi} M_{y} ; c=x_{0}, x, M=\max \left|\frac{1}{G(x)}\right|,
$$

in the rectangle under consideration. Thus, combining (57) and (58): 


$$
\psi_{1}(x)-\psi_{1}\left(x_{0}\right)=R\left\{\frac{i}{\pi} \int_{x_{0}}^{x} \frac{d x}{G(x)}\right\} .
$$

It follows that, if $1 / G(x)$ is regular analytic over the whole interval $(a, b)$, with the possible exception of the end points, (59) holds for any interval $\left(x_{0}, x\right) \subset(a, b)$. Then

$$
p_{1}(x)=R\left\{\frac{i}{\pi} \frac{1}{G(x)}\right\} \quad(a<x<b) \quad\left(d \psi_{1}(x)=p_{1}(x) d x\right) .
$$

We shall illustrate these considerations with the following examples:

(i) $(a, b) \equiv(-1,1) ; p(x)=\left(1-x^{2}\right)^{-1 / 2}$. Here

$$
G(x)=\int_{-1}^{1} \frac{d y}{\left(1-y^{2}\right)^{1 / 2}(x-y)}=\pi\left(x^{2}-1\right)^{-1 / 2} .
$$

The above considerations are applicable and give

$$
p_{1}(x)=R\left\{\frac{i}{\pi} \frac{\left(x^{2}-1\right)^{1 / 2}}{\pi}\right\}=\left(1-x^{2}\right)^{1 / 2} \pi^{-2},
$$

which agrees with the results obtained above (page 81 ).

(ii) $(a, b) \equiv(-1 ; 1) ; p(x)=(1+x)^{\alpha-1}(1-x)^{\beta-1}, \alpha, \beta$ positive integers. Here

$$
\begin{aligned}
& G(x)=\int_{-1}^{1} \frac{(1+y)^{\alpha-1}(1-y)^{\beta-1}}{x-y} d y, \\
& G(x)=Q(x)+(1+x)^{\alpha-1}(1-x)^{\beta-1} \log [(x+1) /(x-1)],
\end{aligned}
$$

where $Q(x)$ is a polynomial with real coefficients, of degree $\leqq \alpha+\beta-3$, or a constant if $\alpha=\beta=1$, which can be written at once by applying the binomial theory to the integrand in (61). Hence, we can again apply (60), with the result

(62) $p_{1}(x)=\frac{(1+x)^{\alpha-1}(1-x)^{\beta-1}}{\left(Q(x)+(1+x)^{\alpha-1}(1-x)^{\beta-1} \log \frac{1+x}{1-x}\right)^{2}+(1+x)^{2(\alpha-1)}(1-x)^{2(\beta-1)} \pi^{2}}$.

\section{Examples:}

$$
\begin{aligned}
& p_{1}(x)=(1+x)^{2} /\left(-4-2 x+(1+x)^{2} \log \frac{1+x}{1-x}\right)^{2}+(1+x)^{4} \pi^{2} \\
& p_{1}(x)=\left[\left(\log \frac{1+x}{1-x}\right)^{2}+\pi^{2}\right]^{-1}[11], \alpha=\beta=1 ;(a, b)=(-1,1)
\end{aligned}
$$


An expression similar to (63) was obtained, though in a different manner and for another purpose, by T. Carleman [11].

(iii) $(a, b) \equiv(-1,1), p(x)=(1+x)^{\alpha-1}(1-x)^{-\alpha}(1>\alpha>0)$. From (36) and (30) we see that in this case $k_{1}=0$, so that (42), (60) yield here

$$
\begin{array}{rlr}
F(x) & =c p(x) & (c=\text { const.), } \\
p_{1}(x) & =1 / p(x) & \text { (within a constant factor). }
\end{array}
$$

This agrees with the result on page 82 , obtained there in an entirely different manner. If, for example, we take $\alpha=\beta=1 / 2,[10]$, then

$$
F(x)=\int_{-1}^{1} \frac{d y}{\left(1-y^{2}\right)^{1 / 2}(x-y)}=\left(x^{2}-1\right)^{-1 / 2},
$$

and consequently by $(65) p_{1}(x)=\left(1+x^{2}\right)^{1 / 2}$ (see page 81$)$.

\section{BIBLIOGRAPHY}

1 Stieltjes, Recherches sur les fractions continues, Oeuvres, vol. 2, pp. 398-566.

${ }^{2}$ O. Perron, Die Lehre von den Kettenbriichen, 2d edition, 1929, Chapters VII, VIII, IX.

${ }^{3} \mathrm{H}$. Hamburger, Ueber eine Erweiterung des Stieltjesschen Momentenproblems, Mathematische Annalen, vol. 81 (1920), pp. 234-319, vol. 82 (1921), pp. 120-164, 168-187.

$1 \mathrm{~J}$. Shohat and J. Sherman, On the numerators ..., Proceedings of the National Academy of Sciences, vol. 18 (1932), pp. 283-287.

5 Jacques Chokhate (J. Shohat), Sur le développement de l'intégrale

Rendiconti di Palermo, vol. 47 (1923), pp. 25-46.

$$
\int_{a}^{b} \frac{p(y) d y}{x-y} \cdots,
$$

- J. Shohat, On the Stieltjes continued fraction, American Journal of Mathematics, vol. 54 (1932), pp. 79-84.

7 Jacques Chokhate (J. Shohat), Sur une classe étendue de fractions continues $\cdots$, Comptes Rendus, vol. 191 (1930), p. 988.

${ }^{8}$ Christoffel, Über die Gaussische Quadratur . . . Crelle, vol. 55 (1858), pp. 61-83, where the differential equation for the Legendre case $\alpha=\beta=1$ is obtained in an entirely different manner.

๑ P. Appell et J. Kampé de Fériet, Fonctions Hypergéométriques et Hypersphériques. Polynomes d'Hermite, Paris, 1926, p. 338.

${ }^{10}$ C. Possé, Sur quelques Applications des Fractions Continues Algébriques, St. Petersbourg, 1886.

$11 \mathrm{~T}$. Carleman, Sur la résolution de certaines équations intégrales, Arkiv för Matematik, Astronomi och Fysik, vol. 16 (1922), No. 26.

University of Pennsylvania, Philadelphia, Pa. 